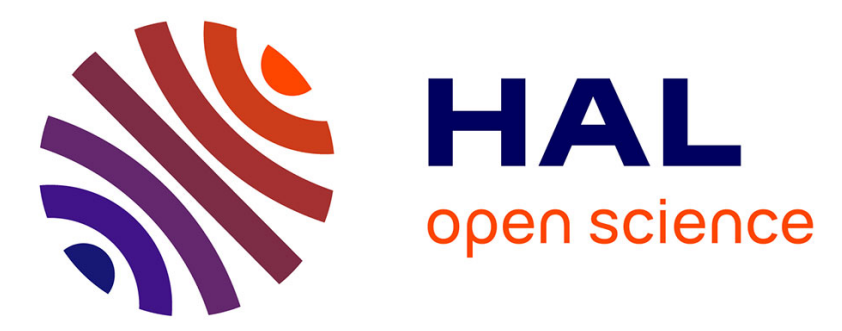

\title{
Catalyst faceting during graphene layer crystallization in the course of carbon nanofiber growth
}

J.-L. Maurice, Didier Pribat, Z He, G Patriarche, Costel Sorin Cojocaru

\section{To cite this version:}

J.-L. Maurice, Didier Pribat, Z He, G Patriarche, Costel Sorin Cojocaru. Catalyst faceting during graphene layer crystallization in the course of carbon nanofiber growth. Carbon, 2014, 79, pp.93. 10.1016/j.carbon.2014.07.047 . hal-01084443

HAL Id: hal-01084443

https://hal-polytechnique.archives-ouvertes.fr/hal-01084443

Submitted on 19 Nov 2014

HAL is a multi-disciplinary open access archive for the deposit and dissemination of scientific research documents, whether they are published or not. The documents may come from teaching and research institutions in France or abroad, or from public or private research centers.
L'archive ouverte pluridisciplinaire HAL, est destinée au dépôt et à la diffusion de documents scientifiques de niveau recherche, publiés ou non, émanant des établissements d'enseignement et de recherche français ou étrangers, des laboratoires publics ou privés.

$$
\text { Copyright }
$$




\title{
Catalyst faceting during graphene layer crystallizationin the course of carbon nanofiber growth
}

\author{
J.-L. Maurice, ${ }^{* a}$ D. Pribat, ${ }^{b}$ Z. He,${ }^{a, c}$ G. Patriarche ${ }^{d}$ and C. S. Cojocaru ${ }^{a}$
}

${ }^{a}$ Laboratoire de Physique des Interfaces et des Couches Minces, LPICM, UMR 7647, CNRSÉcole Polytechnique, Route de Saclay, 91128 Palaiseau Cedex, France.

${ }^{b}$ Department of Energy Science, Sungkyunkwan University, Suwon 440-746, Korea.

${ }^{c}$ State Key Laboratory for Advanced Metals and Materials, University of Science and Technology Beijing, No. 30 Xueyuan Road, Haidian District, Beijing 100083, China.

${ }^{d}$ Laboratoire de photonique et de nanostructures, LPN, UPR 20, CNRS, Route de Nozay, 91460 Marcoussis, France.

The low temperature catalytic growth of multiwall carbon nanotubes (MWCNTs) rests on the continuous nucleation and growth of graphene layers at the surface of crystalline catalystparticles. Here, we study the atomic mechanisms at work in this phenomenon, by observing the growth of such layers in situ in the transmission electron microscope, in the case of iron-based catalysts. Graphene layers, parallel to the catalyst surface, appear by a mechanism of step flow, where the atomic layers of catalyst are "replaced" by graphene planes. Quite remarkably, catalyst facets systematically develop while this mechanism is at work. We discuss the origin of faceting in terms of equilibrium particle shape and graphene layer nucleation. Step bunching due to impeded step migration, in certain growth conditions, yields characteristic catalyst nail-head shapes. Mastering themechanisms of faceting and step bunching could open up the way to tailoring the structure of low temperature-grown MWCNTs, e.g. with highly parallel carbon walls and, ultimately, with controlled structure and chirality.

${ }^{*}$ Corresponding author. E-mail: jean-luc.maurice@ polytechnique.edu (Jean-Luc Maurice) 


\section{Introduction}

Carbon nanofibers (CNFs)[1] or multiwall carbon nanotubes (CNTs, MWCNTs) obtained by catalytic crystallization on metallic particles at low temperature $\left(450-700{ }^{\circ} \mathrm{C}\right)$, have numerous prospects in applications[2-7] but still suffer from irregular - and irreproducible - crystalline structures. The purpose of the present work is to shed some light on the atomic mechanisms that are at work during the crystallization of graphene layers at the surface of iron-based catalysts, to finally propose routes for the low temperature controlled growth of CNTs/CNFs. In the cases of $\mathrm{Ni}[8-10]$ and $\mathrm{Co}[11]$ catalyst nanoparticles, the nucleation and growth of graphene layers has been shown to rely upon the dynamics of surface steps. The growth of MWCNTs with iron-based catalysts has been studied in situ on different scales: in situ XRD experiments[12, 13] have for instance brought important results on the phase of the catalyst during growth. However, studies at the atomic scale have yet brought little data on the role of atomic steps - particularly their dynamics - in the nucleation and growth of graphene.For instance, Rodriguez-Manzo and co-workers[14] have emphasized the role of steps in the nucleation of CNTs from $\mathrm{Co}, \mathrm{Fe}$ and $\mathrm{FeCo}$ (1:1 alloy) nanoparticles using in situ high resolution transmission electron microscopy (HRTEM); however they did not study step flow on the catalysts' surface. Yoshida et al.[15] have observed the growth of MWCNTs on cementite particles $\left(\mathrm{Fe}_{3} \mathrm{C}\right)$ in situ in HRTEM, but did not focus on atomic-scale nucleation of graphene layers. Begtrupet al.[16] have studied Fe faceting during CNT growth, but not in a geometry where step flow was active or visible. Pigoset al. have explained faceting of $\mathrm{Ni}$ catalysts, but during the unsticking of graphene layers rather than during their nucleation[17].

In their seminal paper, Helveget al. have studied Ni-catalyzed CNF growth using in situ environmental transmission electron microscopy (ETEM)[8]. They were the first to clearly show graphene nucleation at step edges on the lateral walls of the catalyst particles. Nucleation was followed by the receding of the metal steps towards the leading and trailing edges of the metal particle, leaving behind a graphene layer. Movements of metallic matter thus promoted graphene growth on the surface terraces of the catalyst and a concomitant elongation of the catalyst particle.

We focus here on the geometry and atomic complexity of those metal steps at the surface of iron-based nanoparticles. We show that, during CNT/CNF growth from Fe-based particles, the movement of these steps tend to create planar facets at the catalyst surface in contact with 
the graphene layer that is being crystallized. Moreover, we find that those steps travel along the curved or faceted surface of the catalyst. Thus, they can adapt themselves to the varying local surface orientation. At the end of their trip along the surface, they may eventually form bunches, which induce some characteristic and familiar shapes of catalysts observed after growth. Mastering this step bunching process could open up the way to a better structural control of CNTs and CNFs grown at low temperature.

\section{Experimental}

The present growth experiments were carried out in situ in the transmission electron microscope (TEM), on catalyst particles around which a nanofiber had already developed during a previous direct-current plasma-enhanced chemical vapor deposition (dc-PECVD) growth in a home-made reactor[18]. Transmission electron microscopy observations were done in a Topcon 002B microscope working at $160 \mathrm{kV}$ (point resolution of $0.2 \mathrm{~nm}$ ). Some in situ experiments were performed in a Philips CM30 instrument working at $300 \mathrm{kV}$ (nominal resolution of $0.23 \mathrm{~nm}$ but $\sim 0.4 \mathrm{~nm}$ in our high temperature observations), using a Gatan Model 652 heating sample holder. Finally, for the in situ HRTEM experiments, we used a Jeol 2200FS microscope working at $200 \mathrm{kV}(0.19 \mathrm{~nm}$ resolution) equipped with a ProtochipsAduro heating sample holder.

In order to dispose of a source of carbon atoms inside the electron microscope, we either amorphized the existing nanotube by electron irradiation [19] or used the carbon present - or injected [14] - in iron carbide $\left(\mathrm{Fe}_{3} \mathrm{C}\right.$, cementite) catalysts. Similar experiments were performed by Rodriguez-Manzoet al. [14] for electron-beam assisted CNT growth inside the TEM, by Ichihashiet al. [20] and Sinclair et al.[21], for growth from an amorphous feedstock, and also by Sun et al. [22], for growth from irradiated iron carbide. Quite interestingly, without prior amorphization, we obtained almost no change when annealing $\alpha$-Fe based nanotubes at $650^{\circ} \mathrm{C}$, while we observed the large changes shown below with $\mathrm{Fe}_{3} \mathrm{C}$, at the lower temperature of $550^{\circ} \mathrm{C}$. We noticed that a part of the carbon atoms dissolved in the cementite catalyst came from the dissolution of graphene planes perpendicular to the catalyst surface (see supplemental material). We had previously observed this phenomenon with $\mathrm{Ni}$ catalysts but not with Fe ones [23].

The in situ experimental protocol was actually the following: 
(i) 30-min irradiation with a focused 300-keV electron beam ( $\alpha-\mathrm{Fe}$ only),

(ii) 10-min annealing at $450^{\circ} \mathrm{C}$,

(iii) 10-min annealing at $550^{\circ} \mathrm{C}$,

(iv) annealing at $650^{\circ} \mathrm{C}(\alpha-\mathrm{Fe}$ only).

We started the heat treatment with the relatively low temperature of $450^{\circ} \mathrm{C}$ because we had observed in another set of experiments using $\mathrm{Ni}$ catalysts that graphene readily crystallized at that temperature during a dcPECVD run[24].

In both cases $-\alpha-\mathrm{Fe}$ and $\mathrm{Fe}_{3} \mathrm{C}$, the electron irradiation at work during observation played a role in the growth process: we did observe changes of shape of the catalyst in reference nonirradiated nanotubes, but of significantly lower extent than in the examples presented below.

We focus here on multiwall carbon nanotubes or nanofibers that have a direct application in actual field emission devices [2]. Direct-current plasma-enhanced chemical vapor deposition allows one to obtain the verticality mandatory for field emission applications [1, 25]. The gas mixture we used for the first growth was made of isopropyl alcohol, water vapor and hydrogen; see details in ref. [18]. We study iron-based catalysts because iron allows one to play with three types of catalysts: $\alpha-\mathrm{Fe}, \gamma-\mathrm{Fe}$ and iron carbides $[13,18]$. Also, compared to nickel, it appears to favor in certain conditions the growth of real MWCNTs (instead of nanofibers), where the graphene layers are parallel to the tube axis [18, 23].

We note that during such a dc-PECVD growth, the CNTs/CNFs have their catalyst particle on top, so that growth beneath the particle remains shielded from the bombardment of incoming plasma species. Moreover, the growth of each new graphene layer is protected from the outside atmosphere by the already formed layers beneath which it develops. Thus the nucleation and growth processes are quite characteristic of the catalyst surface rather than of the details of the gases or plasma conditions. Hence, reproducing a growth experiment in the TEM with no gas (as we do here) remains a valid approach for the general understanding of growth mechanisms, as long as one sticks to the atomic phenomena at work at the catalyst surface.

\section{Results}




\subsection{Ex situ observations}

When observed in the TEM, as-grown CNFs often exhibit features that look like the remnants of a growth by a step flow mechanism. In the CNF with a $\alpha$-Fe catalyst particle shown in Fig. 1 , the start of a new graphene plane always corresponds to a step on the metal surface (blue arrows in Fig. 1b). There exists, moreover, an inversed shoulder beneath the head of the particle (Fig. 1d, yellow circle). The fact that the steps and the shoulder have the same orientationis an indication that the steps have been moving during growth, and that they have somehow gathered to form the inversed shoulder. We shall come back later on this inversed shoulder, leading to a "nail head" shape for the catalyst,which is a distinctive characteristic of PECVD-grown CNTs/CNFs.

The height of the steps at the catalyst surface can hardly be measured directly. However, the disorientation angle $\alpha$ between the terraces and the average catalyst surface should be such that $\tan \alpha=d / D$ where $d$ is the step height and $D$ the terrace width. Here, the latter is worth $10 \mathrm{~nm}$ and the disorientation angle is $\sim 2^{\circ}$ : this gives a step height of $\sim 0.35 \mathrm{~nm}$, i.e. to a good approximation, the interplanar spacing of graphite. It is quite remarkable that the present iron surface, a priori curved around the particle, offer precisely the atomic step height worth the thickness of one graphene layer. Looking in more detail, the local orientation of the present terraces is (33-4). The (33-4) interplanar spacing being $0.05 \mathrm{~nm}$, these atomic planes have a geometrical existence but are not dense enough to have a real physical meaning. Given their height, the present steps include seven such planes. At high magnification (Fig. 1b'), the actual step presents itself like a nanofacet in (110) orientation. Thus the iron surface has arranged itself so as to create steps incommensurate with low-index real Fe atomic planes, but with local nanofacetsfrom which graphene layers appear to be extruded. 


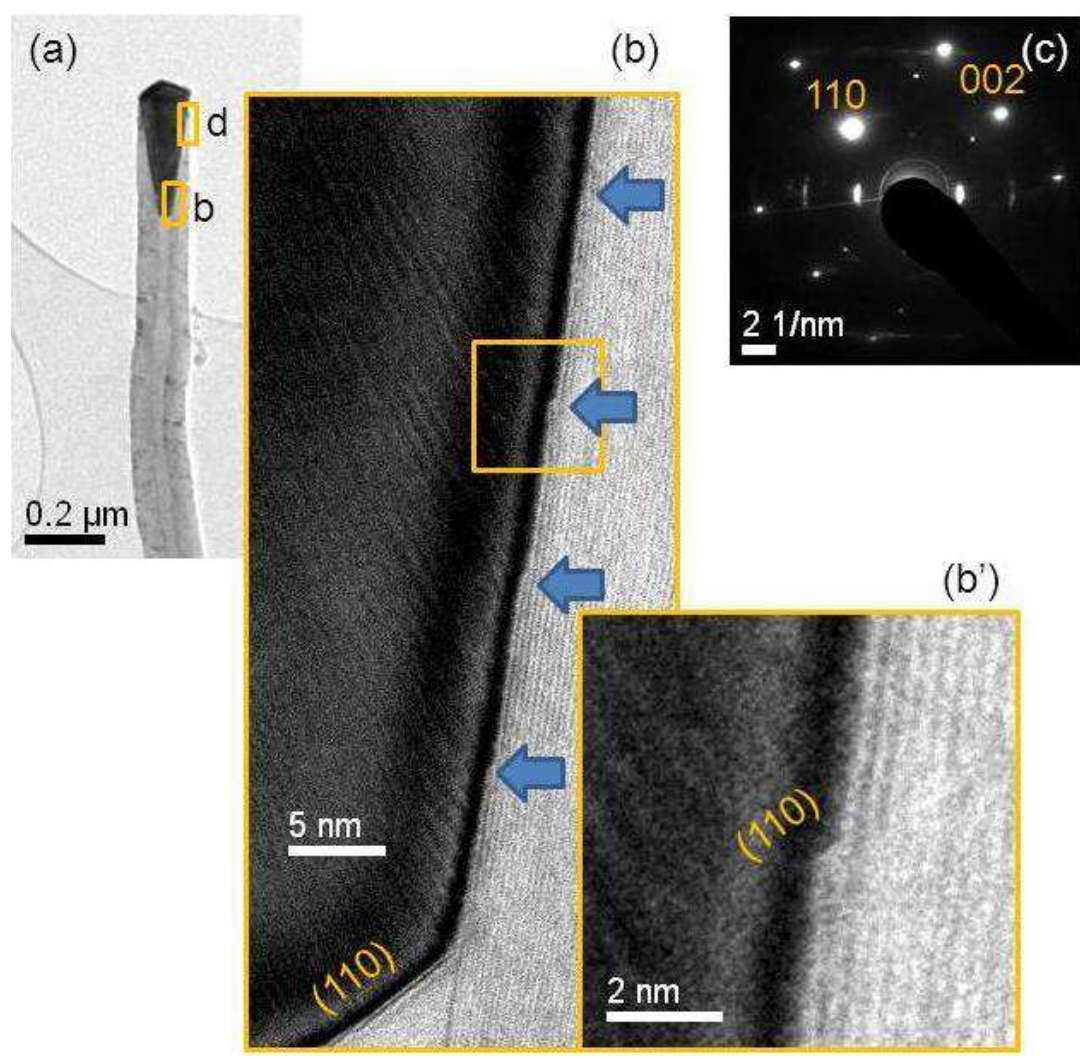

(d)

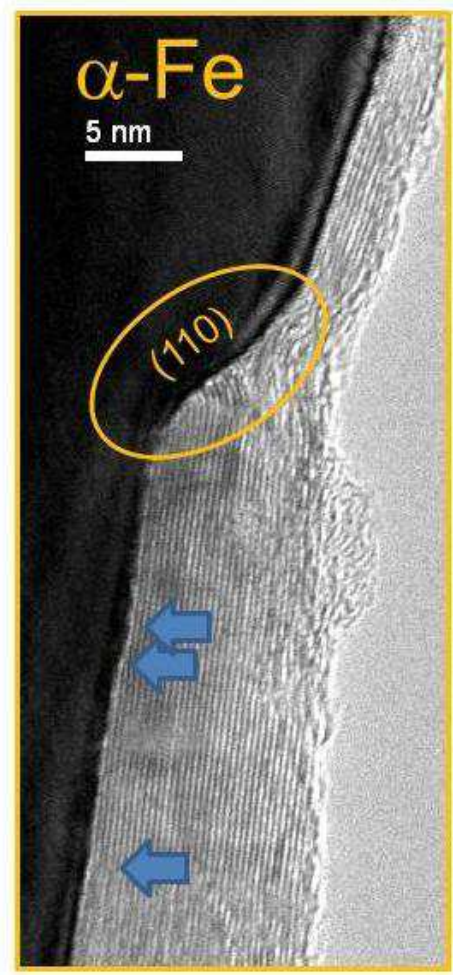

FIG. 1. (Color online) TEM images of a carbon nanofiber obtained with a $\alpha-F e$ catalyst by dcPECVD at $700{ }^{\circ} \mathrm{C}$. a) Low-magnification image; b) and d) high magnification images of respectively the tail and head part of the right-hand side of the catalyst. Blue arrows indicate steps in the catalyst surface that give birth to single graphene layers; b') enlargement of a step in b). (c) Diffraction pattern showing that the catalyst is $\alpha$-Fe in [1-10] zone axis. The (110) indications in (b), (b') and (d) show the tendency of the catalyst to create (110) facets. The circle in d) highlights the inverse shoulder that forms due to step bunching.

Regarding now the step migration and bunching upon heat treatment, they are well known in surface studies: they may be the result of an equilibrium phase transition obtained through surface self-diffusion [26] or they can be part of an irreversible mechanism associated with preferential sublimation at edges [27]; we shall see that the present mechanism is related to self-diffusion. Let us note for the moment that the catalyst steps present two remarkable and original features: $(i)$ they are intimately related to the nucleation and growth of graphene layers, as shown in ref. [8] for a $\mathrm{Ni}$ catalyst and (ii), they have non trivial atomic structure, as they are on a faceted conical surface [28] and their height,although it depends upon the metal 
atomic structure, fitsthe graphite interlayer spacing. This means that the catalyst surface layer that gives birth to a graphene layer by a step-receding mechanismmay include a variable number of catalyst crystallographic planes, depending on the local facet plane.

\subsection{In situ nucleation and growth on an $\alpha$-iron catalyst}

Figure 2 shows the in situ growth at $650^{\circ} \mathrm{C}$ of a proto-nanotube starting from amorphous carbon feedstock [23]. The nanofiber has been amorphized at room temperature by irradiation with a focused $300 \mathrm{keV}$ electron beam [19, 20], during $30 \mathrm{~min}$. Before being annealed at $650^{\circ} \mathrm{C}$, it had firstly undergone in situ heating at $450^{\circ} \mathrm{C}$ and $550^{\circ} \mathrm{C}$, each time for $10 \mathrm{~min}$. A central part of the catalyst particle (which we call sub-nanoparticle in the following) serves as catalyst for the crystallization of the graphene layers of the proto-nanotube. While doing this, it adopts a faceted shape and its volume decreases (the iron atoms moving in the outer parts of the catalyst). Previous observations $[14,16]$ had shown the volume change and the appearance of facets perpendicular to the graphene layers, but this is the first time to our knowledge that faceting parallel to the graphene layers is reported. The shrinking of the subnanoparticle is due to iron atoms migrating away, starting up a creep mechanism that presently leads to forming a sort of corona around the proto-nanotube (see Supplemental material, Fig. S2). It is remarkable that the re-organization of the Fe nanoparticle occurs within a volume that seems to be limited by the original outermost envelope. In particular, the overall diameter of the nanofiber's tip does not evolve during the process, even though there is a large movement of $\mathrm{Fe}$ atoms during the reshaping of the nanoparticle. It looks as if the Fe crystal were now advancing inside the amorphized carbon, incorporating carbon atoms at the $\mathrm{Fe} / \mathrm{C}$ interface, so that this interface moves away from the tip of the nanofiber. 

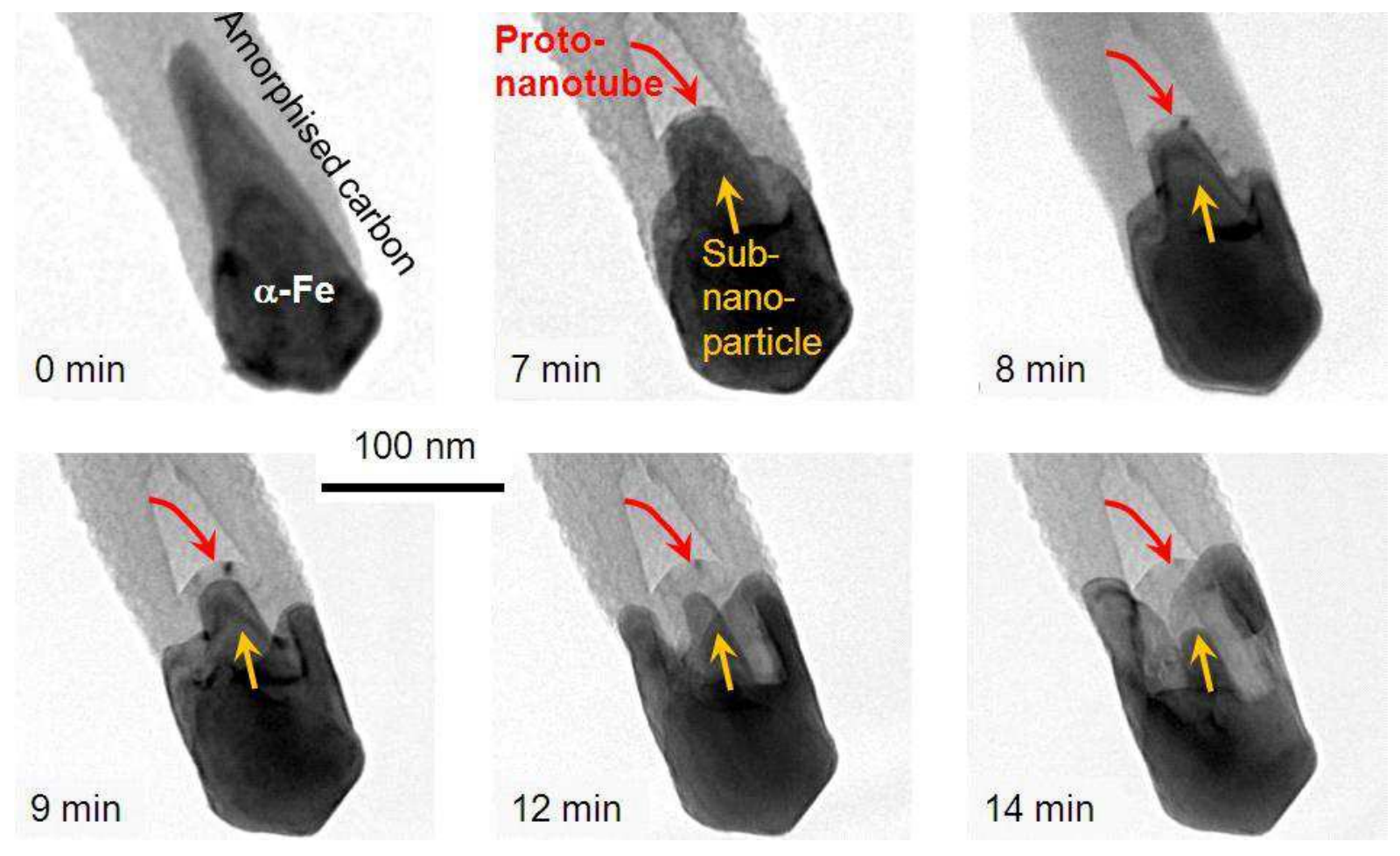

FIG. 2. (Color online) TEM images of a carbon nanofiber firstobtained with $\alpha-F e$ catalyst by dcPECVD at $650^{\circ} \mathrm{C}$ [23]. The fiber has been amorphized at room temperature by irradiation with a focused $300 \mathrm{keV}$ electron beam (see text), then annealed in situinside the TEM at $650^{\circ} \mathrm{C}$ during the time indicated. The arrows are positioned at the same place in all micrographs, to serve as markers. During annealing, the width of the sub-nanoparticle shrinks while the proto-nanotube thickens by its interior, as if Fe atoms in the $\alpha$-Fe lattice were being replaced by $\mathrm{C}$ atoms in the graphene lattice. After $12 \mathrm{~min}$, the shrinkage leads the subnanoparticle to unstick from the protonanotube, which stops the thickening of the latter (see also Supplemental material, Fig. S1).

The outside shape of the catalyst stabilizes, once low index facets have fully developed on the side surface of the Fe nanoparticle (Supplemental material, Fig. S1). The carbon atoms from the amorphous feedstock are incorporated inside the metal (at the advancing $\mathrm{Fe} / \mathrm{C}$ interface) by a ring-shaped surface of the catalyst's head, which progressively becomes the corona (Supplemental material, Figs. S1 \& S2). They may travel to the surface schematized in Fig. 3d 
either following a transport path along the sidewalls of the particle (at the interface with the encapsulating graphene layers) as described by Begtrup[16], or by direct bulk diffusion [14], the carbon diffusion coefficient in $\alpha$-Fe being very high at $650{ }^{\circ} \mathrm{C}$ [18] (See schematic of Cdiffusion in Suppl. material, Fig. S2). While some C atoms have to diffuse all the way up to the tip of the sub-nanoparticle, to feed the nucleation and development of new graphene layers (such as those schematized in Fig. 3d), most of them feed the growth of the already developed external walls of the protonanotube, at the bottom part of the sub-nanoparticle. At 12 min. (Fig. 2), graphene layers start to unstick from the catalyst, which starts in turn to retract, leaving vacuum inside the new nanotube (14 min in Fig. 2). Faceting has disappeared from the tip of catalyst when it is abandoned by the graphene layers; its radius of curvature is $8 \mathrm{~nm}$ at this stage.

Let us now give some details on the structure of the protonanotube and its catalyst. The analysis of the selected-area electron diffraction patterns (EDPs) and their comparison with a real-space image in Fig. 3 indicate that the development of the first graphene layers has induced a $\{110\}$ faceting of the Fe sub-nanoparticle. We note, however, that the two main facets (parallel to the protonanotube axis) are only vicinal to (-101) and (10-1), with a disorientation angle of about $10^{\circ}$ with respect to the [111] zone axis. Given the step height of one $\{110\}$ plane $(0.203 \mathrm{~nm})$, this represents a high density of surface steps: approximately one every $\mathrm{nm}$. We also note, however, that the graphene layers are oriented parallel to the physical surface, and not to the $\{110\}$ planes. Thus the number of steps active in the formation of graphene is probably low. 

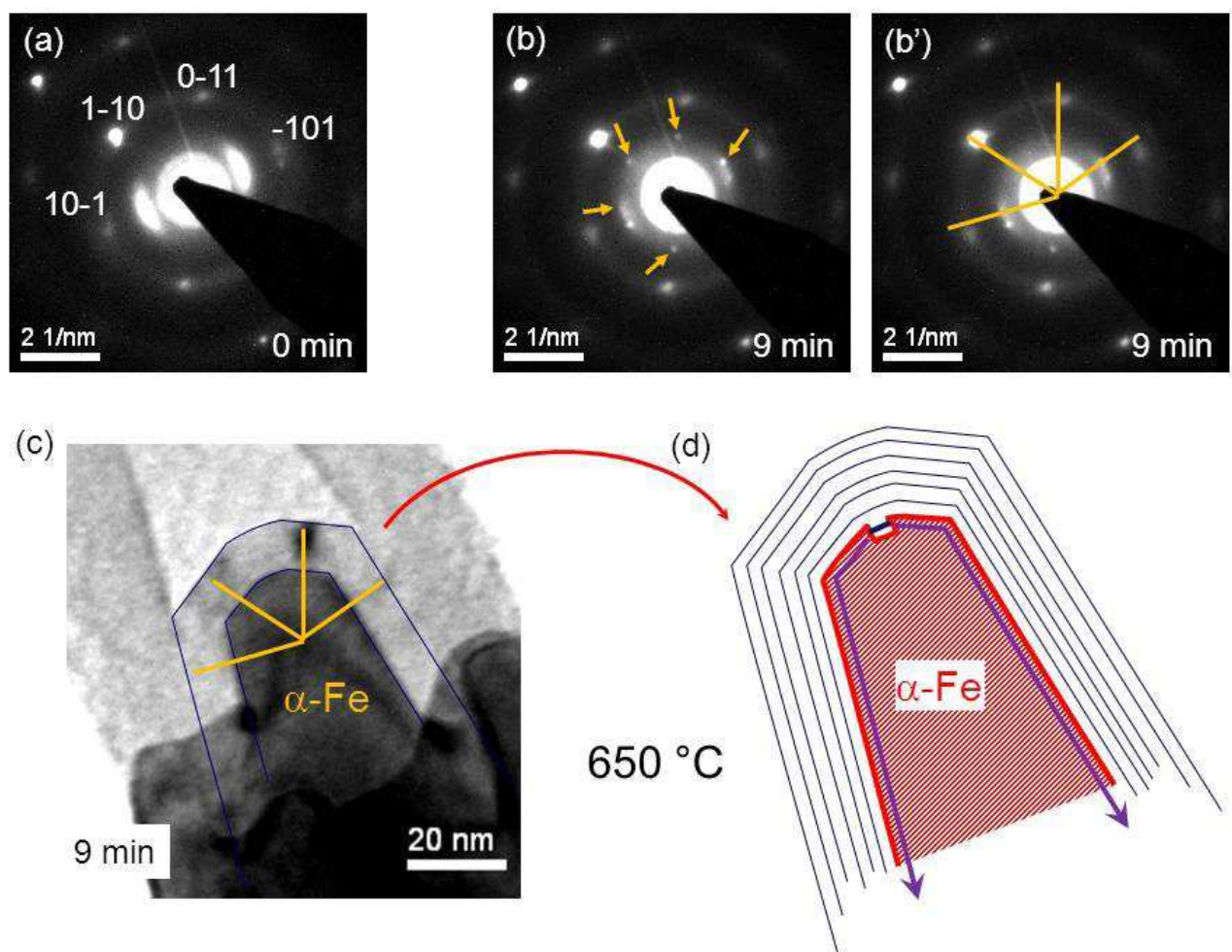

FIG. 3. (Color online) Structure of the protonanotube and catalyst presented in Fig. 2. (a-b) Electron diffraction patterns (EDPs) recorded over an area of $300 \mathrm{~nm}$ around the catalyst: (a) at the beginning of the $650^{\circ} \mathrm{C}$ treatment; (b, b') after 9 min at that temperature (same EDP with different marks). The main reflections in the pattern show that the catalyst particle is a $\alpha-F e$ single crystal viewed in [111] zone axis. These reflections remain unchanged during the 9 min while the particle undergoes the significant change of shape visible in Fig. 2: creep of the particle does not affect its original phase and orientation. After 9 min at $650^{\circ} \mathrm{C}(\mathrm{b}$ and b'), individual 002 graphite reflections become clearly visible (arrows in b), indicating the growth of graphite nanocrystals along preferred orientations: their (002) planes are approximately aligned with the $\{110\}$ iron planes. Drawing these preferred orientations in (b') and reporting them in direct space (image in c), confirms that they correspond ( $i$ ) to a vicinal $\{110\}$ faceting of the Fe catalyst and (ii) to a concomitant faceting of the protonanotube. c, d) Image and corresponding schematic diagram of the protonanotube after 9 min of growth. The correlation between the faceting of the sub-nanoparticle and that of the protonanotube clearly appears. A possible step flow that would allow for the observed catalyst thinning (Fig. 2) is indicated by arrows in d). 
These active steps may be of the type seen in the ex situ experiment (Fig. 1), adapted to the thickness of graphene. However, $\{110\}$ planes in the bcc structureare physically quasi compact and their interplanar spacing is relatively large $(0.203 \mathrm{~nm})$, which makes it difficult to precisely provide a given step height. Moreover, the graphite interlayer spacing $(0.341 \mathrm{~nm})$ has no commensurability with the $\alpha-\mathrm{Fe}\{110\}$ spacing: one needs approximately five $\mathrm{Fe}$ $\{110\}$ planes to accommodate three graphene layers. Thus those active steps might presently correspond to single $\{110\}$ Fe planes andprovoke a disorientation in the graphitestructure like a regular array of partial edge dislocations, the Burgers vector of which would be the difference between the interplanarspacings of $\mathrm{Fe}$ and graphite, i.e. $0.14 \mathrm{~nm}$. Hence, there would be an angle between graphite and Fe surface, due to the presence of these dislocations. Taking a terrace width of about $10 \mathrm{~nm}$ as in Fig. 1, the active steps would provoke a disorientation of the graphene layers with respect to the surface of about 0.8 degrees, which is within the present measurement error. We note that the $\{110\}$ facets have disappeared when the sub-nanoparticle further shrank (Fig. 2, $12 \mathrm{~min}$ and $13 \mathrm{~min}$ ). This transient character would explain why the faceting of the catalyst surface in contact with graphene is often not visible in ex-situ grown samples[29, 30].

Quite remarkably, the large shape evolution of the Fe catalyst implied neither phase change nor orientation shift (see electron diffraction patterns in Fig. 3 a-b). This is another indication that the change in shape is rather governed by surface motion of atoms/steps and not by bulk reorganization such as that observed with smaller catalysts [15]. After 9 min of growth the protonanotube was thus also facetted, with facets parallel to Fe $\{110\}$ facets. Figure $3 d$ gives an example of how a graphene nucleation spot could initiate a step flow responsible for the thinning observed in Fig 2.

In such a scheme of growth, the catalyst surface step and the graphitic plane that it "trails" have to cross facet edges, thus adapting their structure to each new facet orientation. It is important, in this respect to check whether the graphitic planes are indeed continuous across facet edges (see below).

\subsection{Atomic scale observation of nucleation and growth on a cementite catalyst}

In order to get more insights on the way steps and graphene layers cross facet edges, we carried out the annealing experiment in a high-resolution microscope, with carbon nanofibers 
also obtained by dcPECVD at $650{ }^{\circ} \mathrm{C}$ but this time with an iron carbide catalyst ${ }^{\dagger}$. Figure 4 shows lattice-resolved images of the sequential formation of graphene layers at the surface of the cementite particle (for the phase determination, see suppl. material, Fig. S3.). This time, the nutrients for graphene growth are obtained from the catalyst itself, probably through electron-induced injection of carbon atoms[14, 22]. The nanofiber has firstly been annealed in situ for $10 \mathrm{~min}$ at $450^{\circ} \mathrm{C}$. It has then been further annealed in situ for 4 min $10 \mathrm{~s}$ at $550^{\circ} \mathrm{C}$ before the micrograph in Fig. 4a was recorded.

We note that, although cementite has a structure quite different from that of $\alpha$-Fe, the mechanism of graphene layer growth on $\mathrm{Fe}_{3} \mathrm{C}$ exhibits no apparent difference with that on $\alpha$ iron: the active part of the catalyst shrinks and develops facets while it gives birth to successive graphene layers (see suppl. mater. Fig.S4). When focusing on a rounded part of the catalyst (rectangles in Fig. 4 a-a'), we observe that the graphene layers have developed following its curved surface. This would indicate that the catalyst steps at the origin of those layers have traveled across that curved area, following the purple arrow in Fig. $4 \mathrm{~d}$, indicating in turn that the $\mathrm{Fe}_{3} \mathrm{C}$ atomic steps at the origin of graphene layer growth would be of a versatile nature.

As in the case of $\alpha-\mathrm{Fe}$, the catalyst appears to have faceted during graphene layer growth (see suppl. material, Fig. S4). At rounded parts of the catalyst as well as at edges between the facets, most of the graphene layers are continuous (Fig 4). Thus, graphene layers seem to be able to cross these edges during their growth. Such a conclusion is strengthened by the fact that there is no difference in graphene layer numberon either side of facet edges (see Fig. 4c\&e).

\footnotetext{
The starting catalyst is a thin film of pure iron: upon dewetting, a part of it stays in the form of iron nanoparticles when exposed to isopropanol for CNT growth, whereas in the same growth run another part becomes carbide [18].
} 

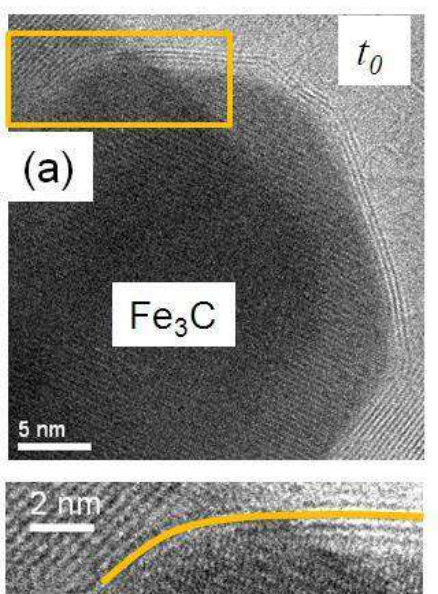

(b)

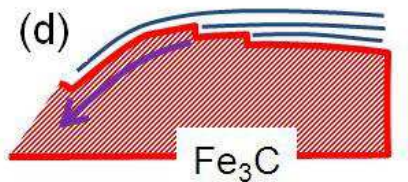

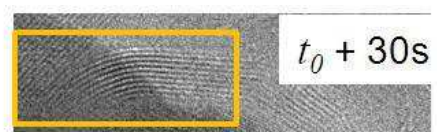
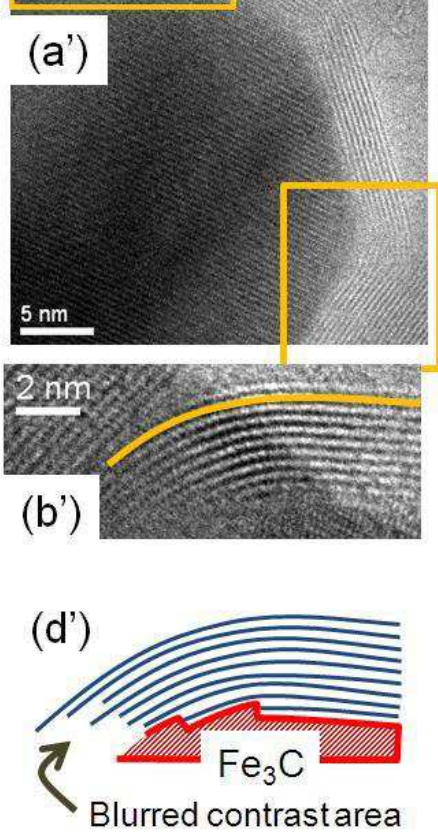

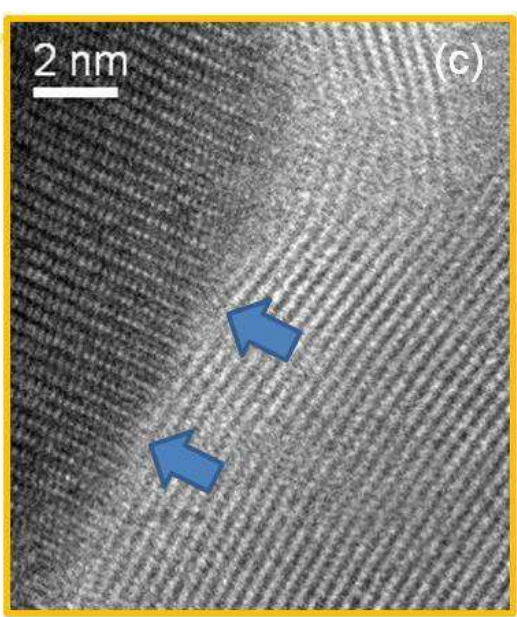

(e)

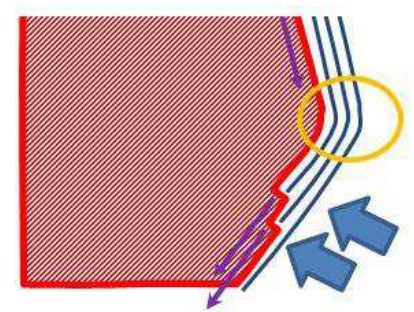

FIG. 4. (Color online) HRTEM images of a carbon nanofiber first obtained with iron carbide catalyst by dcPECVD at $650{ }^{\circ} \mathrm{C}$. In (a) the nanofiber has been annealed in situ for $t_{0}=4 \mathrm{~min} 10 \mathrm{~s}$ at $550^{\circ} \mathrm{C}$. In (a'), after 30 additional seconds at $550^{\circ} \mathrm{C}$, six graphene layers have developed at the expense of an equivalent volume of the carbide catalyst. (b-b' and c) Enlargements of the yellow rectangles in (a-a') showing the surface steps that give birth to the graphene layers; the yellow line in (b-b') is a guide to the eye that shows a same graphene layer before andafter the 30 more seconds. (d-d' and e) Schematic diagrams showing the most likely movement of the steps (purplearrows). The blurred contrast is due to an out-of-focus part of catalyst in the path of the electron beam (see Fig. 5 and supplemental material, Fig. S4, for a view at lower magnification). The blue arrows in (c) and (e) indicate garphene layer edges.

Again here, the faceting of the Fe-based particle evolves as graphene layers are generated, with the presence of steps in facets where graphene layers are "extruded" from the catalyst. In the present case as in that of $\alpha-F e$, the step flow results in a net transport of catalyst matter, which strongly modifies facet surfaces and the catalyst shape in general(see also supplemental material, Fig. S4). Here again, it is quite remarkable that, during such a significant evolution, the catalyst mono-crystalline structure remains apparently unchanged. 


\subsection{Step bunching and collective growth}

As expected when analyzing Fig. 1, the catalyst steps migrate and end up by bunching. This is particularly clear in Fig. 2, where after $12 \mathrm{~min}$ at $650{ }^{\circ} \mathrm{C}$, the protonanotube does not thicken anymore as growth now proceeds by feeding the alreadyexisting graphene layers: all the active steps have gathered at the bottom of the proto nanotube. There is thus a transition from onion-type growth (layer by layer growth by the interior of the nanofiber) to nanotube growth (collective growth of existing graphene layers) that corresponds to the blocking of individual step motion.In the case of our cementite example, step bunching is building up in Fig. 4b' and collective growth is takingplace on the other side of the catalyst during the observations in Fig. 4 (see Supplemental material, Fig. S4). Figure 5 shows a region of the graphite thus obtained in situ after $5 \mathrm{~min} 27 \mathrm{~s}$ at $550^{\circ} \mathrm{C}$. The Fourier transform of the HRTEM image exhibits reflections resembling very much those of standard graphite (Bernal stacking). Thus collective growth has enabled the development of a nanoshell that locally has the graphite structure. 

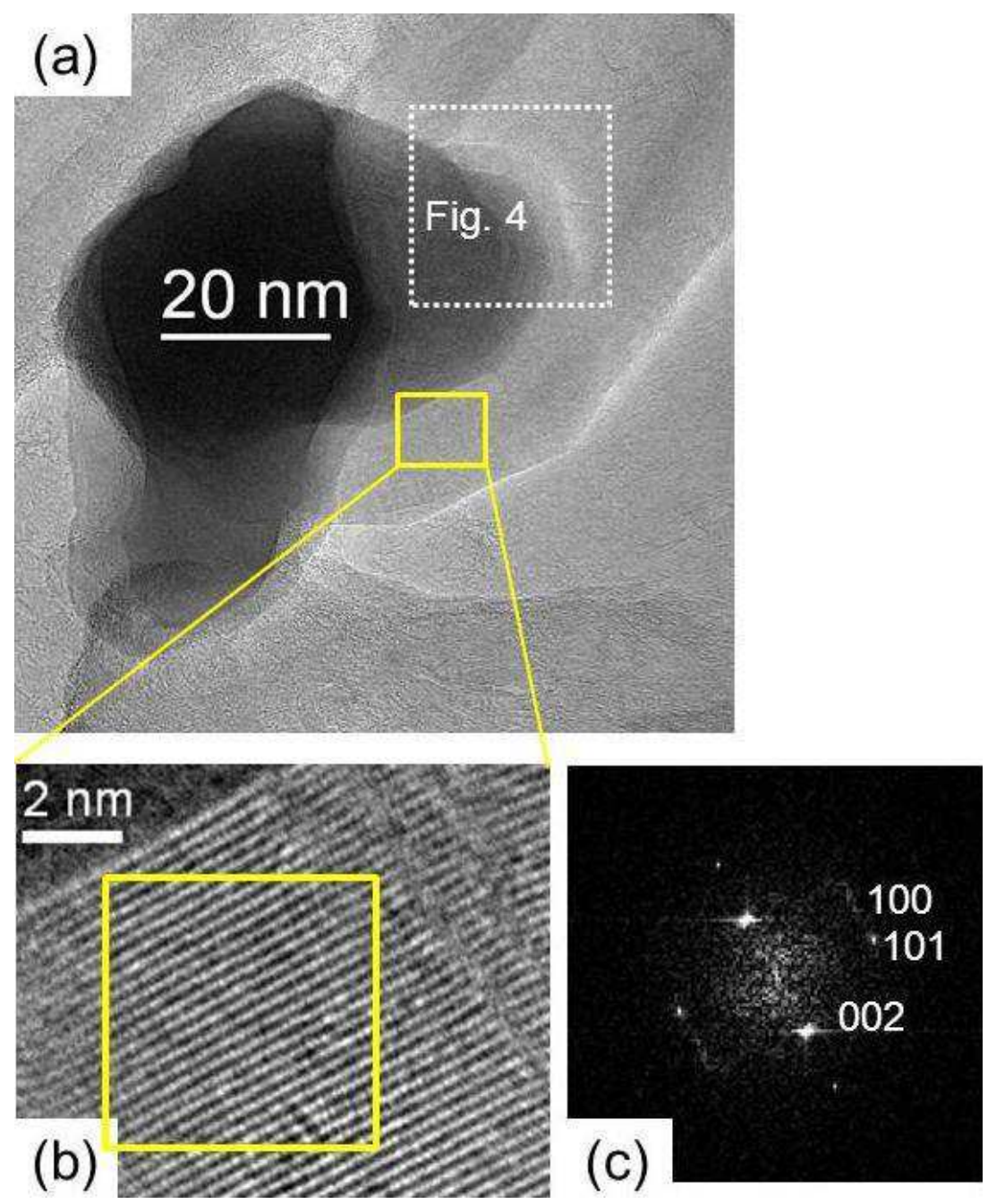

FIG. 5. (Color online) (a) Low magnification image of the cementite catalyst after 5'27 in situ annealing at $550^{\circ} \mathrm{C}$; the dotted square indicates where the images in Fig. 4 have been recorded ( 1 min before the present recordings); the catalyst has been extruded from its original place in the nanotube. (b) HRTEM of the yellow rectangle in (a) showing the graphite structure obtained after collective growth of the graphene layers. The Fourier transform in (c) indicates that the structure in the yellow square in (b) is essentially that of standard graphite.(See suppl. mater. for more information.)

Figure 6 summarizes the two possible geometries of growth. In the present case, the system shifts from individual growth of single graphene layers (onion or nanofiber-type in fig. 6) to the collective growth of all the layers at the same time (nanotube in fig. 6). In the former case, the active interface between catalyst and graphite is parallel to graphene planes; in the latter, it 
is perpendicular (Fig. 6). In the former case, it is a low energy interface(but over a large surface), in the latter, a higher-energy one (but over a small surface)[16].

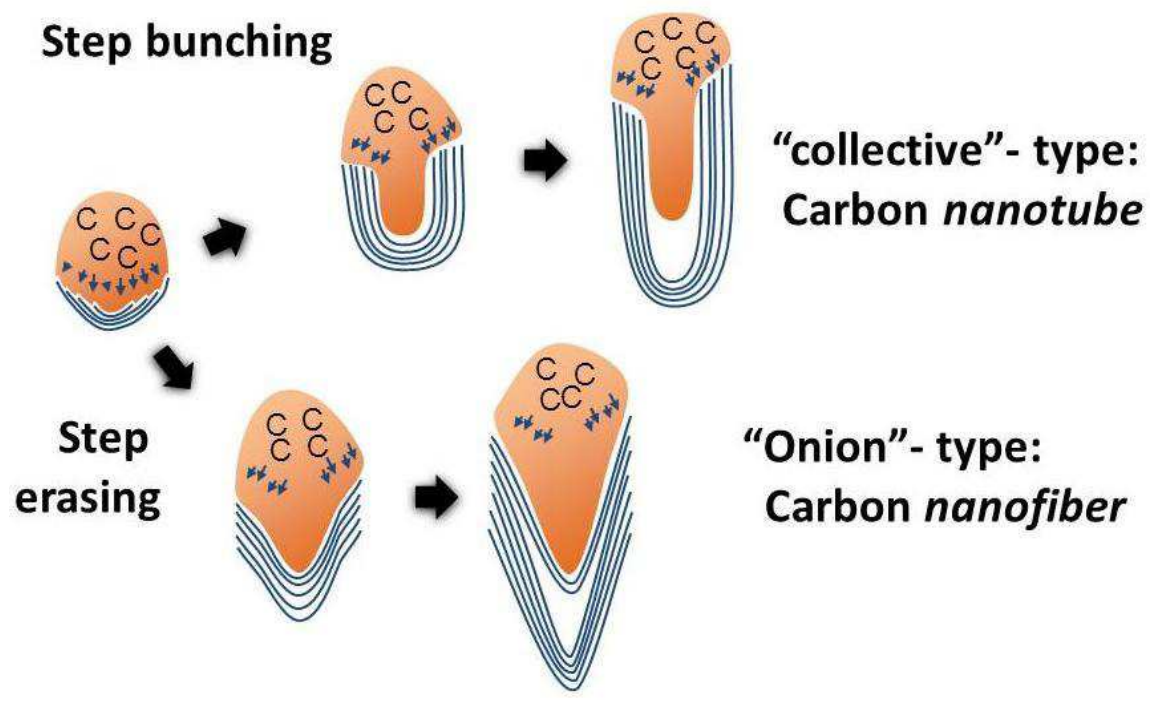

FIG. 6. (Color online) Schematic diagrams showing the two geometries of growth. In the case of collective growth (nanotube, top), catalyst steps are blocked in their progression so that collective growth can take place: no new graphene nucleation events are needed. In the case of onion-like growth (nanofiber, bottom), the step motion brings more matter to the top surface where the steps get "erased": new graphene layers are needed for growth to proceed.

In standard conditions, the dcPECVD growth of nanofibers is essentially of the onion type (Fig. 6) [29]. One sees directly that the electrical current path from bottom to top, in such an object, crosses all the graphene layers. In the case of collective growth of all the layers at the same time on the contrary, the layers end up parallel to the tube axis to form a real multiwall carbon nanotube (Fig. 6). The resistivity of graphite is four orders of magnitude lower in the graphene planes than perpendicular to the planes. Hence, as far as field emission applications are concerned, one sees directly the advantage of being able to tune the growth conditions so as to promote MWCNT growth at the expense of CNF growth.

\section{Discussion: the origin of faceting}


Although faceting of the catalyst has been discussed before [17, 29-31], we are aware of only few previous observations of its occurring during the nucleation of graphene layers[16, 17] and of no previous mention of its occurring parallel to the graphene layers. Furthermore, the present observation of faceting on two quite different types of iron catalysts $(\alpha-\mathrm{Fe}$ and cementite) gives us a clue that this mechanism could be quite general.

We have seen thatcatalyst facets develop while the step flow mechanism, where their atomic planes are "replaced" by graphene layers, is at work. We first discuss whether or not epitaxial relationships between graphene layers and those facets could be a driving force for that development. We start from the (more simple) case of $\alpha$-Fe (Fig. 3). Quite generally, there is no real match between the periodicities of graphene and those of $\alpha-\mathrm{Fe}$. However, the Fe (110) surface provides the less unfavorable epitaxial match between graphene and $\alpha$-iron, with the [10] direction of graphene parallel to the [001] of Fe, and the graphene [12] direction parallel to Fe[1-10] (Fig. 7). Vinogradovet al. have studied thatgeometrical configuration [32] and have found that, in their ultra-high vacuum and stabilized environment, this “epitaxial"relationship was energetically favored. However, several works have focused on the epitaxy of graphene on the a priori more favorable compact surfaces of $\mathrm{Ni}(111)$ and $\mathrm{Co}(001)$. Like $\mathrm{Fe}, \mathrm{Ni}$ and $\mathrm{Co}$ are $3 \mathrm{~d}$ transition metals with an incomplete $\mathrm{d}$ band, likely to offer carbon atoms a bonding similar to that offered by Fe. Those two respective surfaces fit the graphene lattice much better than $\mathrm{Fe}(110)$, with the six-fold symmetry of the graphenelayer and small mismatches of respectively 1.3 and $1.8 \%$. Moreover, these metals are also used for carbon nanotube growth, with results quite similar to those obtained with Fe. In these two more favorable cases, there appears to be a gain in epitaxy, but small.

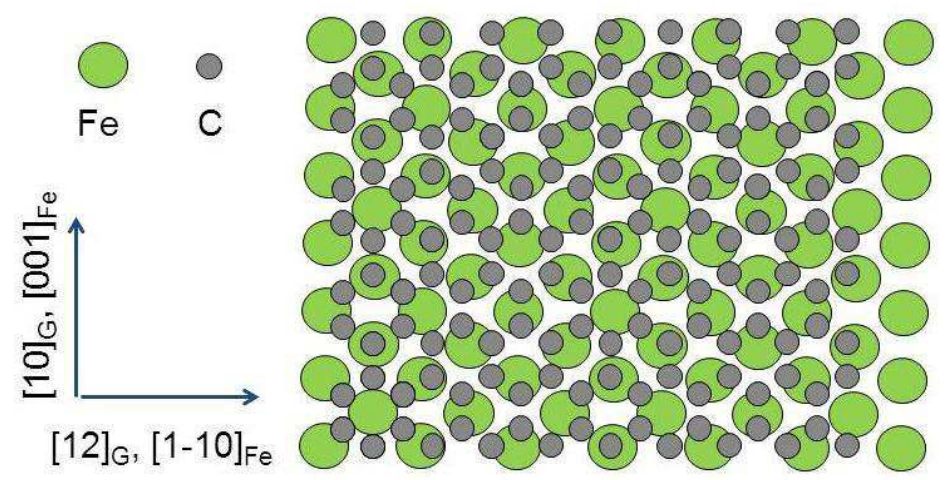

FIG. 7. (Color online) The superimposed structures of graphene and $\alpha$-Fe (110). 
In the case of $\mathrm{Ni}$, Eizenberget al.[33, 34] have noticed that there was "some rotational disorder" about the [111] axis for graphene precipitationon the (111) surface[33], and remarked that "the fact that monolayer condensation is observed on all surfaces studied [i.e. not only (111)] appears to suggest that the nature of the geometrical fit between the overlayer and substrate is of secondary importance". Their experiments were carried out at temperatures between 727 and $827{ }^{\circ} \mathrm{C}$. On our side, we studied the growth of graphene on polycrystalline $\mathrm{Ni}$ after carbon ion implantation and annealing at 725 and $900^{\circ} \mathrm{C}$ : we actually found two mechanisms of growth, including the present step-flow one (which gave the best crystal quality) [35], but could not evidence epitaxial relationships [36].

Concerning cobalt, the C-Co phase diagram is quite similar to that of $\mathrm{C}$ in Ni. Ago et al. have shown that graphene can be epitaxially grown by CVD onto Co (001) at $1000^{\circ} \mathrm{C}$ [37]. However, these authors also showed that growth at a lower temperature of $900^{\circ} \mathrm{C}$ ended up in misaligned graphene, indicating again weak energy gain in the epitaxial relationship.

Thus epitaxy appears as a weak promoter of faceting: the origin of the laterduring graphene layer developmentprobably rests on other causes than simply providing epitaxial match.

A first other cause could be the tendency of any particle to reach its Wulff-polyhedron shape. Having the highest density surface in $\alpha-F e$, bare $\{110\}$ facets have the lowest possible surface energy among the possible free surfaces. They remain the lowest energy facets when considering surfaces covered by one graphene layer [16]. The step flow at catalyst surface, during the development of a graphene layer, generates significant atomic movements in the whole catalyst (see fig. 2, and supplemental material, figs. S1 and S3). These movements are so significant that, if there wasno proof of crystallinity, they could be interpreted as an indication of liquid state. Those movements certainly help the surfaces to reach their lowest energy configuration. Thus areason for the development of low energy $\{110\}$ facets lies in the fact that they become dynamically accessible.

A last cause that must be consideredis the atomic order needed for creating a stable graphene layer nucleus.Yang et al. have recently demonstrated, by producing 92\% single wall carbon nanotubes having the same chirality on WCo catalysts, that a crystalline catalyst 
transmitsindeed a given structure to the growing graphene layer[38]. Abild-Pedersen et al., based on their in situ observations of ref. [8], have carried out a thorough theoretical investigation of the nucleation of graphene layers on nickel, using ab initio calculations [39]. These calculations show that a monoatomic step on the compact $\mathrm{Ni}(111)$ surface is a preferred site for the nucleation of graphene. The energy of creating that step and bringing carbon atoms to develop a graphene nucleusappears as the cheapest way of precipitating a graphene layer. Thus in $\mathrm{Ni}$, nucleationappears favored on the compact surface, even though that includes creating surface steps. A remarkable point is that the step height is the highest for compact surfaces, i.e. the closest to the space needed for the growth of a graphene layer.

The present large Fe-based catalysts are different from the small WCo catalyst of ref [38]probably also by the atomic mobility inside the particle. The catalyst atomic "cradle" in the present case has to move with respect to the rest of the catalyst for the growth to occur: the surface steps glide along the surface plane. As any crystallographic surface can be described in terms of compact-plane nanofacets, it is clear that surfaces along compact planes will be the most appropriate to provide the right plane for that gliding.

Thus low-index facets appear favored nucleation sites for two reasons: $i$ ) they provide the atomic step that has the largest height, the closest to graphite interlayer spacing, and ii) they provide the easiest glide plane for that step.

\section{Conclusions}

The three examples given above are illustrations of the "knitting" of graphite crystal planes by the receding of metal surface steps thatleave behind theircarbon content in the form of graphene layers. The present observations uncover a remarkable property of that phenomenon, which is that it generates faceting of the catalyst, giving a clue that graphene layer nucleation is favored by a given facet step atomic order ..A recent study demonstrates the long-postulated link between catalyst structure and carbon nanotube chirality [38]. The large catalysts used here for the growth of multiwall carbon nanotubes are chemically quite different from the smaller ones involved in ref. [38] but the principle of catalyst atomic order transferred to growing graphene layers has a general nature. The present study shows that, for low temperature growth of MWCNTs, this transfer takes place through catalyst faceting. 
A lastpoint, finally, is that of the end-destination of the catalyst steps. During dcPECVD growth, a "nail head" shape of the catalyst after growth is frequentlyobserved. There, the step migration all around the particle is probably blocked by the ion bombardment on the plasmaexposed surface, leading to step bunching which creates the shoulder (Figs. 1c and 6top). Note that in this situation, a first stage of growth must involve the step flow discussed here, which thins down the bottom part of the catalyst. In a second stage however, no new graphene layers are created, only those already existing continue to grow, eventually leading to the geometry of real MWCNT. In such a geometry, where graphene layers are parallel to the tube axis[18], all the graphite planes making up the nanotube now grow at the same time.

In the framework of applications involving electrical conductivity, like the field emission of interest here, that geometry should drastically decrease the nanotube resistance as conductivity along the planes in graphite is four orders of magnitude better than across the planes.

\section{Acknowledgements}

JLM \& CSC would like to acknowledge fruitful discussions with Dr P. Legagneux, Dr JP.Mazellier and F. Andrianiazy (Thales R\&T France) as well as with Dr I. Florea (LPICM). JLM \& ZH thank Dr. G. Garry (Thales R\&T France) for the use of the Topcon 002 microscope, as well as Dr . G. Rizza and Dr . P.-E. Coulon (Laboratoire des SolidesIrradiés, Ecole Polytechnique, France), for the use of the CM 30 microscope. ZH and JLM thank Dr A. Gohier (then at LPICM) for providing samples. JLM and GP thank B. Jacobs and S. Shannon (Protochips, inc.) for their help with the Aduro holder. This work has been partly supported by French ANR in the framework of the Equipex program TEMPOSNanoMAX. 
[1] Melechko AV, Merkulov VI, McKnight TE, Guillorn MA, Klein KL, Lowndes DH, et al. Vertically aligned carbon nanofibers and related structures: Controlled synthesis and directed assembly. J Appl Phys. 2005;97(4).

[2] Teo KBK, Minoux E, Hudanski L, Peauger F, Schnell J-P, Gangloff L, et al. Microwave devices: Carbon nanotubes as cold cathodes. Nature. 2005;437(7061):968-.

[3] Bokobza L. Multiwall carbon nanotube elastomeric composites: A review. Polymer. 2007;48(17):4907-20.

[4] Zhang LD, Fang M. Nanomaterials in pollution trace detection and environmental improvement. Nano Today. 2010;5(2):128-42.

[5] Ren XM, Chen CL, Nagatsu M, Wang XK. Carbon nanotubes as adsorbents in environmental pollution management: A review. Chem Eng J. 2011;170(2-3):395-410.

[6] Haniu H, Saito N, Matsuda Y, Tsukahara T, Usui Y, Narita N, et al. Basic potential of carbon nanotubes in tissue engineering applications. Journal of Nanomaterials. 2012;2012:343747.

[7] Schnorr JM, Swager TM. Emerging applications of carbon nanotubes. Chem Mater. 2010;23(3):646-57.

[8] Helveg S, López-Cartes C, Sehested J, Hansen PL, Clausen BS, Rostrup-Nielsen JR, et al. Atomic-scale imaging of carbon nanofibre growth. Nature. 2004;427:426-9.

[9] Hofmann S, Sharma R, Ducati C, Du G, Mattevi C, Cepek C, et al. In situ observations of catalyst dynamics during surface-bound carbon nanotube nucleation. Nano Lett. 2007;7(3):602-8.

[10] Lin M, Tan JPY, Boothroyd C, Loh KP, Tok ES, Foo YL. Dynamical observation of bamboo-like carbon nanotube growth. Nano Lett. 2007;7(8):2234-8.

[11] Zhu HW, Suenaga K, Hashimoto A, Urita K, Hata K, Iijima S. Atomic-resolution imaging of the nucleation points of single-walled carbon nanotubes. Small. 2005;1(12):11803.

[12] Cambedouzou J, Landois P, Rouzière S, Pinault M, Mocuta C, Hennet L, et al. Anomalous thermal expansion of $\gamma$-iron nanocrystals inside multiwalled carbon nanotubes. Phys Rev B. 2013;88(8):081402.

[13] Wirth CT, Bayer BC, Gamalski AD, Esconjauregui S, Weatherup RS, Ducati C, et al. The phase of iron catalyst nanoparticles during carbon nanotube growth. Chem Mater. 2012;24(24):4633-40. 
[14] Rodriguez-Manzo JA, Terrones M, Terrones H, Kroto HW, Sun LT, Banhart F. In situ nucleation of carbon nanotubes by the injection of carbon atoms into metal particles. Nature Nanotechnol. 2007;2(5):307-11.

[15] Yoshida H, Takeda S, Uchiyama T, Kohno H, Homma Y. Atomic-scale in-situ observation of carbon nanotube growth from solid state iron carbide nanoparticles. Nano Lett. 2008;8(7):2082-6.

[16] Begtrup GE, Gannett W, Meyer JC, Yuzvinsky TD, Ertekin E, Grossman JC, et al. Facets of nanotube synthesis: High-resolution transmission electron microscopy study and density functional theory calculations. Phys Rev B. 2009;79(20):205409.

[17] Pigos E, Penev ES, Ribas MA, Sharma R, Yakobson BI, Harutyunyan AR. Carbon nanotube nucleation driven by catalyst morphology dynamics. ACS Nano. 2011;5(12):10096101.

[18] He Z, Maurice J-L, Gohier A, Lee CS, Pribat D, Cojocaru CS. Iron catalyst for the growth of carbon nanofibers: Fe, Fe3C or both? Chem Mater. 2011;23(24):5379-87.

[19] Krasheninnikov AV, Banhart F. Engineering of nanostructured carbon materials with electron or ion beams. Nat Mater. 2007;6(10):723-33.

[20] Ichihashi T, Fujita J-I, Ishida M, Ochiai Y. In situ observation of carbon-nanopillar tubulization caused by liquidlike iron particles. Phys Rev Lett. 2004;92(21):215702.

[21] Sinclair R, Itoh T, Chin R. In situ TEM studies of metal-carbon reactions. Microsc Microanal. 2002;8(4):288-304.

[22] Sun L, Banhart F, Krasheninnikov AV, Rodríguez-Manzo JA, Terrones M, Ajayan PM. Carbon nanotubes as high-pressure cylinders and nanoextruders. Science. 2006;312(5777):1199-202.

[23] Maurice JL, He ZB, Cojocaru CS. Different mechanisms of graphene wall nucleation on Fe and Ni particles. Advances in Imaging and Electron Physics. 2013;179:142-4.

[24] Lee CS, Cojocaru CS, Moujahid W, Lebental B, Chaigneau M, Châtelet M, et al. Synthesis of conducting transparent few-layer graphene directly on glass at $450^{\circ} \mathrm{C}$. Nanotechnology. 2012;23(26):265603.

[25] Chhowalla M, Teo KBK, Ducati C, Rupesinghe NL, Amaratunga GAJ, Ferrari AC, et al. Growth process conditions of vertically aligned carbon nanotubes using plasma enhanced chemical vapor deposition. J Appl Phys. 2001;90(10):5308-17.

[26] Williams ED, Bartelt NC. Surface faceting and the equilibrium crystal shape. Ultramicroscopy. 1989;31(1):36-48. 
[27] Wilson N, Marsden A, Saghir M, Bromley C, Schaub R, Costantini G, et al. Weak mismatch epitaxy and structural Feedback in graphene growth on copper foil. Nano Res. 2013;6(2):99-112.

[28] He Z, Ke X, Bals S, Van Tendeloo G. Direct evidence for the existence of multiwalled carbon nanotubes with hexagonal cross-sections. Carbon. 2012;50(7):2524-9.

[29] He ZB, Maurice J-L, Lee CS, Gohier A, Pribat D, Legagneux P, et al. Etchant-induced shaping of nanoparticle catalysts during chemical vapour growth of carbon nanofibres. Carbon. 2011;49:435-44.

[30] He ZB, Maurice J-L, Lee CS, Cojocaru CS, Pribat D. Nickel catalyst faceting in plasma-enhanced direct current chemical vapor deposition of carbon nanofibers. AJSE. 2010;35(1C):19-28.

[31] Tessonnier J-P, Su DS. Recent progress on the growth mechanism of carbon nanotubes: A review. ChemSusChem. 2011;4(7):824-47.

[32] Vinogradov NA, Zakharov AA, Kocevski V, Rusz J, Simonov KA, Eriksson O, et al. Formation and structure of graphene waves on Fe(110). Phys Rev Lett. 2012;109(2):026101.

[33] Eizenberg M, Blakely JM. Carbon monolayer phase condensation on Ni(111). Surf Sci. 1979;82(1):228-36.

[34] Eizenberg M, Blakely JM. Carbon interaction with nickel surfaces: Monolayer formation and structural stability. J Chem Phys. 1979;71:3467-77.

[35] Baraton L, He ZB, Lee CS, Cojocaru CS, Châtelet M, Maurice JL, et al. On the mechanisms of precipitation of graphene on nickel thin films. EPL (Europhys Lett). 2011;96(4):46003.

[36] Baraton L, He Z, Lee CS, Maurice J-L, Cojocaru CS, Gourgues-Lorenzon A-F, et al. Synthesis of few-layers graphene by ion implantation of carbon in nickel thin films. Nanotechnology. 2011;22:085601.

[37] Ago H, Ito Y, Mizuta N, Yoshida K, Hu B, Orofeo CM, et al. Epitaxial chemical vapor deposition growth of single-layer graphene over cobalt film crystallized on sapphire. ACS Nano. 2010;4(12):7407-14.

[38] Yang F, Wang X, Zhang D, Yang J, LuoDa, Xu Z, et al. Chirality-specific growth of single-walled carbon nanotubes on solid alloy catalysts. Nature. 2014;510(7506):522-4.

[39] Abild-Pedersen F, Norskov JK, Rostrup-Nielsen JR, Sehested J, Helveg S. Mechanisms for catalytic carbon nanofiber growth studied by ab initio density functional theory calculations. Phys Rev B. 2006;73(11):115419. 